\title{
Bone Marrow-on-a-Chip Replicates Hematopoietic Niche Physiology in Vitro
}

\section{Citation}

Torisawa, Yu-suke, Catherine S. Spina, Tadanori Mammoto, Akiko Mammoto, James C. Weaver, Tracy Tat, James J. Collins, and Donald E. Ingber. 2014. Bone Marrow-on-a-Chip Replicates Hematopoietic Niche Physiology in Vitro. Nature Methods 11, no. 6: 663-669.

\section{Published Version}

doi:10.1038/nmeth.2938

\section{Permanent link}

http://nrs.harvard.edu/urn-3:HUL.InstRepos:12872207

\section{Terms of Use}

This article was downloaded from Harvard University's DASH repository, and is made available under the terms and conditions applicable to Other Posted Material, as set forth at http:// nrs.harvard.edu/urn-3:HUL.InstRepos:dash.current.terms-of-use\#LAA

\section{Share Your Story}

The Harvard community has made this article openly available.

Please share how this access benefits you. Submit a story.

\section{Accessibility}




\section{Bone marrow-on-a-chip replicates hematopoietic niche physiology in vitro}

Yu-suke Torisawa ${ }^{1 *}$, Catherine S. Spina ${ }^{1,2^{\star}}$, Tadanori Mammoto ${ }^{3}$, Akiko Mammoto $^{3}$, James C. Weaver ${ }^{1}$, Tracy Tat ${ }^{3}$, James J. Collins ${ }^{1,2,4,5}$, and Donald E. Ingber ${ }^{1,3,6}$

${ }^{1}$ Wyss Institute for Biologically Inspired Engineering, Harvard University, Boston, MA 02115; ${ }^{2}$ Boston University School of Medicine, Boston, MA 02118; ${ }^{3}$ Vascular Biology Program, Departments of Pathology and Surgery Children's Hospital Boston and Harvard Medical School; ${ }^{4}$ Howard Hughes Medical Institute, ${ }^{5}$ Department of Biomedical Engineering, Boston University, Boston, MA 02115, USA; ${ }^{6}$ School of Engineering and Applied Science, Harvard University, Cambridge, MA 02138, USA; *Both authors contributed equally.

Running title: Bone marrow-on-a-chip

Key words: hematopoietic stem cells, bone marrow transplantation, regeneration, tissue engineering, microfluidics, organs-on-chips

Address correspondence to: Donald E. Ingber, M.D., Ph.D.

Wyss Institute for Biologically Inspired Engineering at Harvard University

CLSB5, 3 Blackfan Circle

ph: $617-432-7044$

fax: 617-432-7828

em: don.ingber@wyss.harvard.edu 
Current in vitro hematopoiesis models fail to demonstrate the cellular diversity and complex functions of living bone marrow and hence, most translational studies relevant to the hematologic system are conducted in live animals. Here, we describe a method for fabricating a "bone marrow-on-a-chip" that permits culture of a living marrow with a functional hematopoietic niche in vitro by first engineering new bone in vivo, removing it whole, and perfusing it with culture medium in a microfluidic device. The engineered marrow retains hematopoietic stem and progenitor cells in normal in vivo-like proportions for at least one week in culture. The device models organ-level marrow toxicity responses and protective effects of radiation countermeasure drugs, whereas conventional bone marrow culture methods do not. This biomimetic microdevice offers a new approach for analysis of drug responses and toxicities in bone marrow, as well as study of hematopoiesis and hematologic diseases in vitro.

The bone marrow microenvironment contains a complex set of cellular, chemical, structural and physical cues necessary to maintain the viability and function of the hematopoietic system ${ }^{1-5}$. This hematopoietic niche regulates hematopoietic stem cells (HSCs), facilitating a delicate balance between self-renewal and differentiation into progenitor cells that produce all mature blood cell types ${ }^{4,5}$. Engineering an artificial bone marrow that reconstitutes natural marrow structure and function, and that can be maintained in culture, could be a powerful platform to study hematopoiesis and test new therapeutics. It has proven difficult, however, to recreate the complex bone marrow microenvironment needed to support the formation and maintenance of a complete, functional hematopoietic niche in vitro ${ }^{6-9}$. Although various in vitro culture systems have 
been developed to maintain and expand hematopoietic stem and progenitor cells ${ }^{6-11}$, there is currently no method to recreate or study the intact bone marrow microenvironment in vitro. Therefore, studies on hematopoiesis commonly rely on animal models to ensure the presence of an intact bone marrow microenvironment that enables normal physiologic marrow responses ${ }^{12-15}$. Furthermore, although it has been reported that bone marrow can be engineered in vivo ${ }^{16-19}$, no method exists to culture engineered bone marrow in vitro. To bridge the functional gap between in vivo and in vitro systems, we developed a method to produce a "bone marrow-on-a-chip" culture system that contains artificial bone and a living marrow, which is first generated in mice, and then explanted whole and maintained in vitro within a microfluidic device.

\section{RESULTS}

\section{In vivo engineering of bone marrow}

Tissue engineering methods have been used to induce formation of new bone with a central marrow compartment in vivo ${ }^{18-21}$. To explore the possibility of engineering an artificial bone marrow that can be explanted whole, we microfabricated a polydimethylsiloxane (PDMS) device with a central cylindrical cavity $(1 \mathrm{~mm}$ high $\times 4 \mathrm{~mm}$ diameter) with openings at both ends (Fig. 1a). We filled the hollow compartment with a type I collagen gel containing bone-inducing demineralized bone powder (DBP) and bone morphogenetic proteins (BMP2 and BMP4) $)^{20-22}$, and implanted it subcutaneously on the back of a mouse (Supplementary Fig. 1). Our goal was to engineer bone that would fill the cylindrical space within the implanted device so that it could be easily removed whole and inserted into a microfluidic system containing a similarly shaped 
chamber for in vitro culture (Fig. 1 a,b). These initial studies resulted in the creation of new bone encasing a marrow compartment that formed within the PDMS device 4 to 8 weeks after subcutaneous implantation. Histological analysis revealed that the marrow was largely inhabited by adipocytes and exhibited a low level of hematopoietic cell contribution, even 8 weeks after implantation (Fig. 1C), as previously noted by others using similar tissue engineering approaches with bone-inducing materials ${ }^{19-21}$.

The presence of large numbers of adipocytes in bone marrow can inhibit hematopoiesis $^{23}$. To reduce adipocyte content in the marrow, we sealed the top of central cavity in the implanted device by adding a solid layer of PDMS to restrict access of cells or soluble factors from the overlying adipocyte-rich hypodermis to the boneinducing materials, while maintaining accessibility to the underlying muscle tissue through the lower opening (Fig. 1a, Supplementary Fig. 1). Subcutaneous implantation of this improved PDMS device resulted in the formation of a cylindrical disk of white, boney tissue containing a central blood-filled marrow over a period of 8 weeks (Fig. 1b, Supplementary Fig. 2). Histological analysis confirmed the presence of a shell of cortical bone of relatively uniform thickness surrounding a marrow dominated by hematopoietic cells, containing only rare adipocytes (Fig. 1c). Comparison of histological sections of the engineered bone marrow (eBM) to sections from an intact femur confirmed that the morphology of the eBM was nearly identical to that of natural bone marrow (Fig. 1c).

Micro-computed tomographic (micro-CT) analysis of the eBM demonstrated that the newly formed cortical shell of bone also contained an ordered internal trabecular network that closely resembles the intricate architecture found in normal adult mouse 
vertebrae (Supplementary Fig. 3 ), and which is known to be supportive of $\mathrm{HSCs}^{24}$

(Fig. 1d). Compositional analysis using energy dispersive x-ray spectroscopy (EDS) also showed that the calcium and phosphorus content of the eBM are indistinguishable from natural trabecular bone (Supplementary Fig. 3).

\section{Characterization of engineered bone marrow}

Interactions between CXCL12 expressed on the surfaces of various cell types in the bone marrow (e.g., osteoblasts ${ }^{25}$, perivascular endothelial and perivascular stromal cells $^{26}$ ) and its cognate receptor CXCR4 on the surfaces of HSCs and hematopoietic progenitor cells are critical for the recruitment, retention and maintenance of $\mathrm{HSCs}^{26-28}$. Immunohistochemical analysis confirmed that both of these key hematopoietic regulators are expressed in their normal positions in the eBM: CXCL12 localized to cells lining the inner surface of the bone and blood vessels, while CXCR4 was expressed by clusters of lymphoid cells in the endosteal and perivascular niches (Fig. 2 a-d). We also confirmed that key hematopoietic niche cells ${ }^{29}$ including perivascular Nestin+ cells and Leptin receptor+ cells as well as CD31+ vascular endothelial cells reside in their normal positions (Supplementary Fig. 4). Together, these data demonstrate that our modified bone tissue engineering strategy produced a cortical shell of bone with an internal network of trabecular bone and a normal appearing marrow filling its central space. The presence of key cellular and molecular components of the hematopoietic niche suggests that the cellular content of the eBM closely resembles that of natural bone.

To rigorously characterize the hematopoietic content of the engineered marrow, cells were harvested from the eBM immediately after surgical removal and analyzed by 
flow cytometry. The cellular components of the marrow contained within the eBM were compared to hematopoietic populations isolated from femur bone marrow and peripheral blood from the same mice (Fig. 2 e,f). Devices harvested 4 and 8 weeks after implantation contained all blood cell types, including HSCs $\left(\mathrm{Lin}^{-} \mathrm{Sca} 1^{+}{ }^{+} \mathrm{CKit}^{+} \mathrm{CD} 34^{+/-}\right.$, Lin ${ }^{-S c a} 1^{+} \mathrm{CKit}^{+} \mathrm{CD} 150^{+/-} \mathrm{CD} 48^{-/+}$), and hematopoietic progenitor cells identified by four different marker sets $\left(\operatorname{Lin}^{-} \mathrm{Sca} 1^{+}, \mathrm{Lin}^{-} \mathrm{CKit}{ }^{+}, \mathrm{Lin}^{-} \mathrm{CD} 34^{+}, \operatorname{Lin}^{-} \mathrm{CD} 135^{+}\right)$, as well as mature erythrocytes (Ter119), lymphocytes ( $\mathrm{T}$ cells, $\mathrm{CD} 45^{+} \mathrm{CD} 3^{+} ; \mathrm{B}$ cells, $\mathrm{CD} 45^{+} \mathrm{CD} 19^{+}$) and myeloid cells $\left(\mathrm{CD} 45^{+} \mathrm{Mac1}^{+/-} \mathrm{Gr} 1^{+/-}\right)$. The eBM harvested 4 weeks after implantation did not appear to be fully developed, as indicated by a lower proportion of HSCs and hematopoietic progenitor cells, compared to normal marrow (Fig. 2 g,h). However, cells harvested from the eBM 8 weeks after implantation exhibited a completely normal distribution of HSCs, hematopoietic progenitors, and differentiated blood cells from all lineages that was nearly identical to that displayed by natural bone marrow (Fig. $\mathbf{2} \mathbf{~ g , h}$ and Supplementary Fig. 5, 6). Together, these data demonstrate that the eBM consists of a cylindrical disk of cortical and trabecular bone (Supplementary Fig. 3) containing marrow that also has nearly identical hematopoietic cell composition as natural bone marrow.

\section{In vitro culture of engineered bone marrow}

To determine whether the eBM can maintain a functional hematopoietic system in vitro, the eBM formed 8 weeks after implantation was surgically removed from the mouse, punctured in multiple places with a surgical needle to permit fluid access, and cultured in another clear PDMS microfluidic device containing a similarly shaped, cylindrical central chamber that is separated from overlying and underlying microfluidic 
channels by porous membranes (Fig. 1 a,b). To maintain cellular viability of the eBM, culture medium was perfused through the top and bottom channels using a syringe pump at a rate $(1 \mu \mathrm{L} / \mathrm{min})$ optimized to maintain cellular viability (Supplementary Fig. 7) once being inserted into the central chamber and the surrounding porous membranes and microchannel layers were attached. The eBMs were cultured in vitro for 4 or 7 days within the bone marrow chip microsystem (Fig. 1b), which covers a time period that is commonly used to test for drug efficacies and toxicities in vitro ${ }^{30,31}$. The cultured bone and marrow retained their morphology during this time, including the distribution of CXCL 12-expressing stromal cells_(Supplementary Fig. 8). Stroma-supported culture systems represent the current benchmark for maintaining survival of HSCs and hematopoietic progenitor cells in vitro ${ }^{7,32}$. Thus, we used flow cytometric analysis to compare the hematopoietic cellular composition of the cultured bone marrow-on-a-chip to that of marrow isolated from mouse femur cultured for the same amount of time on a stromal 'feeder' cell layer (Supplementary Fig. 9). Because past work has shown that addition of cytokines is required to maintain or expand HSCs and their progenitors ${ }^{6,7}$, and serum can suppress the marrow reconstituting activity of $\mathrm{HSCs}^{33}$, the stromasupported cultures were maintained in serum-free medium supplemented with cytokines (mSCF, mlL-11, mFLt-3 ligand, and hLDL) that have been shown by others to more efficiently maintain and expand both HSCs and hematopoietic progenitor cell populations in vitro ${ }^{34}$. Our analysis revealed that there was no significant difference in cell viability after 4 or 7 days of culture in the microfluidic eBM device compared to the static stroma-supported culture (Supplementary Fig. 10). However, bone marrow cultured on stroma exhibited a significant decrease $(P<0.0005)$ in the number of long- 
term HSCs ( Lin $^{-} \mathrm{CD} 150^{+} \mathrm{CD}^{-} 8^{-}$cells $)$and a concomitant increase $(p<0.0005)$ in hematopoietic progenitor cells ( Lin $^{-} \mathrm{CD} 34^{+}, \mathrm{Lin}^{-} \mathrm{Sca} 1^{+}, \mathrm{Lin}^{-} \mathrm{CKit}^{+}$) relative to cells freshly isolated from natural mouse bone marrow (Fig. $\mathbf{3}$ a,b). Thus, the long-term HSCs, which are the only cells capable of long-term self-renewal and multi-lineage potential, appear to be differentiating into more specialized progenitor cells in the static stromasupported cultures system, as previously reported ${ }^{6-9}$. In contrast, the number and distribution of HSCs and hematopoietic progenitor cells in the eBM cultured for up to 7 days on-chip were maintained in similar proportions to freshly harvested bone marrow (Fig. 3a). Importantly, the bone marrow-on-a-chip enabled maintenance of a significantly higher proportion of long-term HSCs, while more effectively maintaining the distribution of mature blood cells compared to the stroma-supported cultures (Fig. 3b). Interestingly, while the proportions of hematopoietic cells were retained over this culture period, there was no significant difference in the number or viability of cells cultured onchip for 7 days compared to 4 days, hence the HSCs and hematopoietic progenitor cells appeared to remain relatively quiescent in the marrow-on-a-chip microdevice. Moreover, while addition of exogenous (and expensive) cytokines, including mSCF, mlL-11, mFLt-3 ligand, and hLDL are critical for maintenance of these cell populations in conventional stroma-supported cultures ${ }^{6,7,34}$, their removal from culture medium had little effect on the distribution of HSCs and hematopoietic progenitors in the cultured eBM (Fig. 3d). Thus, the eBM contains a functional hematopoietic niche that behaves in an autonomous fashion to support the continued survival of these critical bloodforming stem and progenitor cells in vitro. Taken together, these data suggest that the bone marrow-on-a-chip contains a functional hematopoietic microenvironment that 
supports and regulates blood cell populations in normal proportions that can be maintained for at least one week under microfluidic flow in vitro, even in the absence of exogenous cytokines.

To confirm that the HSCs and hematopoietic progenitor cells retained in the cultured bone marrow-on-a-chip remain truly functional, we evaluated their self-renewal and differentiation capabilities by testing engraftment and hematopoietic reconstitution potential following transplantation into lethally-irradiated, syngeneic, recipient mice. Cells within the marrow compartments of eBMs that were formed in GFP-expressing animals and cultured on-chip for 4 days were harvested and transplanted into $\gamma$ irradiated mice; results were compared to irradiated mice that were transplanted with cells freshly harvested bone marrow from mouse femur. Total engraftment was assessed in the peripheral blood of recipient mice 6 and 16 weeks after transplantation to confirm the presence of functional short- and long-term HSCs, respectively. Cells harvested from the eBM following 4 days in culture on-chip successfully engrafted the mice at a similar rate as freshly isolated, uncultured bone marrow (Fig. 3e), and repopulated all differentiated blood cell lineages (Fig. 3f) showing $70 \%$ and $85 \%$ engraftment by 6 and 16 weeks after transplantation, respectively. These data confirm that the hematopoietic compartment of the eBM retains fully functional, self-renewing, multi-potent HSCs after being cultured in the microfluidic bone marrow chip for 4 days in vitro.

\section{In vitro model for radiation toxicity}

The functionality and organ-level responsiveness of the bone marrow-on-a-chip were tested by exposing the eBM to varying doses of $\gamma$-radiation to determine whether 
this method can be used as an in vitro model for radiation toxicity, which currently can only be studied in live animals. Live mice, eBMs cultured on-chip, and marrow cells maintained in stroma-supported culture were exposed to 1 and 4 Gray (Gy) doses of $\gamma$ radiation that have been previously shown to produce marrow toxicity in mice ${ }^{35}$, and maintained in culture. When measured 4 days after radiation exposure, we detected a statistically significant, radiation dose-dependent decrease in the proportion of HSCs (Fig. 4a), hematopoietic progenitors (Fig. 4 b,c), lymphoid cells (Fig. 4d), and myeloid cells (Fig. 4e), which closely mimics what is observed in the bone marrow of live, irradiated mice. Interestingly, the proportion of HSCs $\left(\mathrm{LinSca}^{-}{ }^{+} \mathrm{CKit}^{+}\right)$or progenitors $\left(\operatorname{Lin}^{-} \mathrm{CD}_{34}{ }^{+}\right)$observed in eBM after exposure to 1 and 4 Gy doses of $\gamma$-radiation were nearly identical to the proportions measured in whole marrow from live mice that underwent similar irradiation. In contrast, the proportion of HSCs and progenitors were significantly $(P<0.05)$ lower in the stroma-supported culture after 4 Gy irradiation compared to 1 Gy irradiation. Various types of marrow cells (HSCs, progenitors, lymphoid and myeloid cells) cultured on stroma also exhibited suppressed responses, and all were significantly $(P<0.05)$ more resistant to the effects of radiation toxicity (Fig 4 a-e, Supplementary Fig. 11).

To further evaluate the functional relevance and power of our system, we tested the effects of administering granulocyte colony-stimulating factor (G-CSF), which has been shown to accelerate recovery and prevent potentially lethal bone marrow failure following radiation-exposure in vivo ${ }^{36}$. When G-CSF was added to the eBM cultured onchip one day after exposure to $\gamma$-radiation, samples analyzed three days later demonstrated a significant increase in the total number of HSCs $\left(\mathrm{Lin}^{-} \mathrm{Sca} 1^{+} \mathrm{CKit}^{+}\right)$and 
hematopoietic progenitor cells ( Lin $\left.^{-} \mathrm{CKit}^{+}, \mathrm{Lin}^{-} \mathrm{CD} 34^{+}\right)$compared to untreated bone marrow chips that were similarly irradiated (Fig. 4f). These findings suggest that GCSF induced proliferation of HSCs and hematopoietic progenitor cells in the bone marrow chip in vitro, as previously reported in vivo ${ }^{36}$. These data clearly demonstrate that the bone marrow-on-a-chip faithfully mimics the natural physiological response of living bone marrow to clinically relevant doses of $\gamma$-radiation and to a validated radiation countermeasure drug (G-CSF), whereas conventional stroma-supported cultures do not.

\section{DISCUSSION}

Our bone marrow-on-a-chip fabrication strategy provides an important proof-of-concept for the creation of an organ-on-chip device that reconstitutes and sustains an intact, functional, living bone marrow when cultured in vitro. This strategy differs significantly from conventional tissue engineering approaches in which materials or living cells are implanted in vivo without geometric constraint, and without any intent of removing the newly formed organ and maintaining its viability ex vivo. While we regenerate the complex structural, physical and cellular microenvironment of whole bone marrow by employing in vivo tissue engineering techniques, we then leverage microfluidic strategies to deliver nutrients, chemicals and other soluble signals in a fashion that supports the continued viability and function of this engineered organ in vitro. This also differs from most organ-on-chip approaches that use microengineering and microfluidics approaches to model tissue architecture, cell-cell relationships, chemical gradients and 
the mechanical microenvironment, and then inhabit the devices with cultured cell lines or isolated stem cells ${ }^{37}$.

Our in vivo engineering approach enabled us to reconstitute hematopoietic niche physiology and restore complex tissue-level functions of natural bone marrow. The eBM autonomously produces the factors necessary to support the maintenance and function of the hematopoietic system in vitro, which offers a major practical advantage over existing culture systems in that expensive growth supplements can be removed from the culture medium or greatly reduced. Another advantage is that the bone marrow-on-a-chip supports hematopoietic stem and progenitor cells in normal in vivolike proportions relative to the other hematopoietic cell populations and maintains their spatial positions within a fully formed 3D bone marrow niche in vitro. These features of the bone marrow-on-a-chip are likely key to its ability to preserve complex functionalities of the whole organ that cannot be replicated by conventional stroma-supported cultures. Importantly, the use of microfluidics also enables analysis of responses under flow, which is important for both regulation of marrow physiology $5,6,10,38,39$ and study of pharmacokinetic and pharmacodynamic (PK/PD) behaviors of drugs that are critical for evaluation of their clinical behavior.

The eBM cultured on-chip mimics complex tissue-level responses to radiation toxicity normally only observed in vivo, and to a therapeutic countermeasure agent (GCSF) that is known to accelerate recovery from radiation-induced toxicity in patients ${ }^{40}$. Thus, this biomimetic microsystem could serve as a valuable in vitro replacement for whole animals in the testing and development of drugs and other medical countermeasures that might protect against radiation poisoning in the future. 
Apparently, the completeness of our organ mimic permits us to recapitulate the physiologic responses of the whole hematopoietic niche to clinically relevant cues (e.g., cytokines, drugs, radiation) whereas conventional cell cultures do not. This finding underscores the novelty of maintaining a functional marrow containing multiple components of the hematopoietic niche in vitro, rather than merely culturing particular hematopoietic cell types.

The bone marrow-on-a-chip provides an interesting alternative to animal models, because it offers the unique ability to manipulate individual hematopoietic cell populations (e.g., genetically or using drugs), or to insert other cell types (e.g., tumor cells) in vitro, before analyzing the response of the intact marrow to relevant clinical challenges, including radiation or pharmaceuticals. It also might be possible to generate human bone marrow models; for example, an eBM could be engineered in immunocompromised mice (e.g., NOD.Cg-Prkdc $c^{\text {scid }} \| 2 \mathrm{rg}^{\text {tm1 } 1 \text { Wil }} / \mathrm{Sz}$; NSG) that have their endogenous marrow cells replaced with human hematopoietic cells.

The ability to produce trabecular bone with architectural and compositional properties similar to natural bone offers a way to produce bones of pre-defined size and shape, and it could represent a new method to study bone biology, remodeling, and bone pathophysiology in vitro. Therefore, the bone marrow-on-a-chip is a powerful method to accelerate discovery and development in a wide range of biomedical fields ranging from hematology, oncology, and drug discovery to tissue engineering. 


\section{Acknowledgements}

We thank G.Q. Daley for guidance and helpful discussions, and P.L. Wenzel, N. Arora, E. Jiang, A. Jiang, B. Mosadegh, D. Huh, A. Bahinski, and G.A. Hamilton for their technical assistance and advice. This work was supported by the Wyss Institute for Biologically Inspired Engineering at Harvard University, the Defense Advanced Research Projects Agency under Cooperative Agreement Number W911NF-12-2-0036, and the U.S. Food and Drug Administration (FDA) HHSF223201310079C.

\section{Author contributions}

Y.T., C.S.S. and D.E.I. conceived the experiments; Y.T. and C.S.S. performed the experiments, designed research and analyzed data with assistance from T.M., A.M., J.C.W., T.T., J.J.C., and D.E.I. Y.T., C.S.S., and D.E.I. wrote the manuscript. 


\section{REFERENCES}

1. Sacchetti, B. et al. Self-renewing osteoprogenitors in bone marrow sinusoids can organize a hematopoietic microenvironment. Cell 131, 324-336 (2007).

2. Chan, C. K. F. et al. Endochondral ossification is required for haematopoietic stemcell niche formation. Nature 457, 490-494 (2009).

3. Mendez-Ferrer, S. Mesenchymal and haematopoietic stem cells form a unique bone marrow niche. Nature 466, 829-834 (2010).

4. Orkin, S.H. \& Zon LI. Hematopoiesis: An evolving paradigm for stem cell Biology. Cell 132, 631-644 (2008).

5. Wang, L. D. \& Wagers, A. J. Dynamic niches in the origination and differentiation of haematopoietic stem cells. Nat. Rev. Mol. Cell Biol. 12, 643-655 (2011).

6. Maggio, N. D. et al. Toward modeling the bone marrow niche using scaffold-based 3D culture systems. Biomaterials 32, 321-329 (2011).

7. Takagi, M. Cell processing engineering for ex-vivo expansion of hematopoietic cells. J. Biosci. Bioeng. 99, 189-196 (2005).

8. Nichols, J. E. et al. In vitro analog of human bone marrow from 3D scaffolds with biomimetic inverted colloidal crystal geometry. Biomaterials 30, 1071-1079 (2009).

9. Cook, M. M. et al. Micromarrows-three-dimensional coculture of hematopoietic stem cells and mesenchymal stromal cells. Tissue Eng. 18, 319-328 (2012).

10. Csaszar, E. et al. Rapid expansion of human hematopoietic stem cells by automated control of inhibitory feedback signaling. Cell Stem Cell 10, 218-229 (2012).

11. Boitano, A. E. et al. Aryl hydrocarbon receptor antagonists promote the expansion of human hematopoietic stem cells. Science 329, 1345-348 (2010). 
12. Cao, X. et al. Irradiation induces bone injury by damaging bone marrow microenvironment for stem cells. Proc. Natl. Acad. Sci. USA 108, 1609-1614 (2011).

13. Askmyr, M., Quach, J. \& Purton, L. E. Effects of the bone marrow microenvironment on hematopoietic malignancy. Bone 48, 115-120 (2011).

14. Greenberger, J. S. \& Epperly, M. Bone marrow-derived stem cells and radiation response. Semin. Radiat. Oncol. 19, 133-139 (2009).

15. Medas, M. B., Hazlehurst, L. A. \& Dalton, W. S. The bone marrow microenvironment as a tumor sanctuary and contributor to drug resistance. Clin. Cancer Res. 14, 2519$2526(2008)$.

16. Scotti, C. et al. Engineering of a functional bone organ through endochondral ossification. Proc. Natl. Acad. Sci. USA 110, 3997-4002 (2013).

17. Lee, J. et al. Implantable microenvironments to attract hematopoietic stem/cancer cells. Proc. Natl. Acad. Sci. USA 109, 19638-19643 (2012).

18. Reddi, A. H. \& Huggins, C. Biochemical sequences in the transformation of normal fibroblasts in adolescent rats. Proc. Nat. Acad. Sci. USA 69, 1601-1605 (1972).

19. Krupnick, A., Shaaban, S., Radu, A. \& Flake, A. W. Bone marrow tissue engineering. Tissue Eng. 8, 145-155 (2002).

20. Chen, B. et al. Homogeneous osteogenesis and bone regeneration by demineralized bone matrix loading with collagen-targeting bone morphogenetic protein-2. Biomaterials 28, 1027-1035 (2007).

21. Schwartz, Z. et al. Differential effects of bone graft substitutes on regeneration of bone marrow. Clin. Oral. Impl. Res. 19, 1233-1245 (2008). 
22. Ekelund, A., Brosjo, O. \& Nilsson, O. S. Experimental induction of heterotopic bone. Clin. Orthop. Relat. Res. 263, 102-112 (1991).

23. Naverias, O. et al. Bone-marrow adipocytes as negative regulators of the haematopoietic microenvironment. Nature 460, 259-263 (2009).

24. Xie, Y. et al. Detection of functional haematopoetic stem cell niche using real-time imaging. Nature 457, 97-101 (2009).

25. Calvi, L. M. et al., Osteoblastic cells regulate the haematopoietic stem cell niche. Nature 425, 841-846 (2003).

26. Ding, L. \& Morrison, S. J. Haematopoietic stem cells and early lymphoid progenitors occupy distinct bone marrow niches. Nature 495, 231-235 (2013).

27.Zou, Y.-R., et al., Function of the chemokine receptor CXCR4 in haematopoiesis and in cerebellar development. Nature 393, 595-599 (1998).

28. Peled, A., et al., The chemokine SDF-1 stimulates integrin-mediated arrest of CS34(+) cells on vascular endothelium under shear flow. J. Clin. Invest. 104, 1199$1211(1999)$.

29. Mendez-Ferrer, S., et al., Mesenchymal and hematopoietic stem cells form a unique bone marrow niche. Nature 466, 829-834.

30. Olaharski, A. J. et al., In vitro to in vivo concordance of a high throughput assay of bone marrow toxicity across a diverse set of drug candidates. Toxicol. Lett. 188, 98103 (2009).

31. Hoeksema, K.A. et al., Systematic in-vitro evaluation of the $\mathrm{NCI} / \mathrm{NIH}$ Developmental Therapeutics Program Approved Oncology Drug Set for the identification of a 
candidate drug repertoire for MLL-rearranged leukemia. OncoTargets and Therapy 4, 149-168 (2011).

32. Dexter, T. M., Wright, E. G., Krizsa, F. \& Lajtha, L. G. Regualtion of haemopoietic stem cells proliferation in long term bone marrow cultures. Biomedicine. 27, 344-349 (1977).

33. Bryder, D. \& Jacobsen, E. W. Interleukin-3 supports expansion of long-term multilineage repopulating activity after multiple stem cell divisions in vitro. Blood 96, 1748-1755 (2000).

34. Miller, C. L. \& Eaves, C. J. Expansion in vitro of adult murine hematopoietic stem cells with transplantable lympho-myeloid reconstituting ability. Proc. Natl. Acad. Sci. 94, 13648-13653 (1997).

35. Williams, J. P. et al., Animal models for medical countermeasures to radiation exposure. Radiat. Res. 173, 557-578 (2010).

36. Cary, L. H., Ngudiankama, B. F., Salber, R. E., Ledney, G. D. \& Whitnall, M. H. Efficacy of radiation countermeasures depends on radiation quality. Radiat. Res. $177,663-675(2012)$.

37. Huh, D. et al., Microengineered physiological biomimicry: organs-in-chips. Lab Chip 12, 2156-2164 (2012).

38. Schwartz, R. M., Palsson, B. O. \& Emerson, S. G. Rapid medium perfusion rate significantly increase the productivity and longevity of human bone marrow cultures. Proc. Natl. Acad. Sci. USA 88, 6760-6764 (1991). 
39. Wendt, D., Stroebel, S., Jakob, M., John, G. T. \& Martin, I. Uniform tissues engineered by seeding and culturing cells in 3D scaffolds under perfusion at defined oxygen tensions. Biorheol. 43, 481-488 (2006).

40. Herodin, F. \& Drouet, M. Cytokine-based treatment of accidentally irradiated victims and new approaches. Exp. Hematol. 33, 1071-1080 (2005). 


\section{FIGURE LEGENDS}

Fig. 1. In vivo bone marrow engineering. (a) Workflow to generate a bone marrow-on-a-chip system in which engineered bone marrow (eBM) is formed in a PDMS device in vivo and is then cultured in a microfluidic system. (b) Photographs showing the PDMS device containing bone-inducing materials in its central cylindrical chamber before implantation (top panel), the formed white cylindrical bone with pink marrow visible within eBM 8 weeks after implantation (middle panel), and the bone marrow chip microdevice used to culture the eBM in vitro (lower panel) (bar, $2 \mathrm{~mm}$ ). (c) Low (left) and high (right) magnification views of histological H\&E-stained sections of the eBM formed in the PDMS device with two opening (top), one lower opening (middle) at 8 weeks following implantation compared with a cross-section of bone marrow within the normal adult mouse femur (bottom) (bars, 50 and $500 \mu \mathrm{m}$ for low and high magnification views, respectively). (d) Three-dimensional reconstruction of microcomputed tomography (micro-CT) data from eBM 8 weeks after implantation (average bone volume was $2.95 \pm 0.25 \mathrm{~mm}^{3} ; \mathrm{n}=3$ ).

Fig. 2. Localization of cytokines and hematopoietic cell composition of the engineered bone marrow. (a-d) Immunohistochemical analysis of ligand-receptor pair, CXCL12 and CXCR4, in eBM compared to mBM (uncultured, mouse femur bone marrow). Anti-CXCL12 staining of the eBM (a) and mBM (b). Anti-CXCR4, staining of eBM (c) and $\mathrm{mBM}(\mathbf{d})$. Images are taken at 63X magnification (bars, $25 \mu \mathrm{m})$. (e) Flow cytometric analysis of HSCs and hematopoietic progenitor cells within the Lin negative cell subpopulation isolated from $\mathrm{mBM}$ and $\mathrm{eBM}$ isolated 8 weeks after subcutaneous implantation. Numbers inside individual gates indicate the proportion of these cells as a 
percentage of the total cell population isolated from whole bone marrow.

Distribution of HSCs (Lin`Sca $1^{+}{ }^{-} \mathrm{CKit}^{+}$, red) and hematopoietic progenitor cells (Lin-Sca1 ${ }^{+}$,

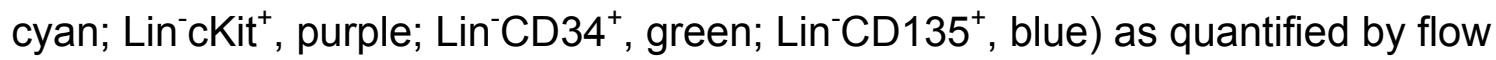
cytometric analysis of $\mathrm{mBM}(n=6)$, eBM at $4(n=5)$ or 8 weeks $(n=5)$ after implantation, or mouse peripheral blood $(\mathrm{mPB})(n=1)$ that underwent erythrocyte lysis to facilitate detection of rare HSCs. Flow cytometry plots (f) and distribution (h) of matured, lineage-restricted cell types including erythrocytes $\left(\operatorname{Ter} 119^{+}\right.$, blue), myeloid cells (CD45 ${ }^{+} \mathrm{Mac}^{+}$, orange; $\mathrm{CD} 45^{+} \mathrm{Gr} 1^{+}$, green; $\mathrm{CD} 45^{+} \mathrm{Mac} 1^{+} \mathrm{Gr} 1^{+}$, purple), B cells $\left(\mathrm{CD} 45^{+} \mathrm{CD} 19^{+}\right.$, cyan) and T cells $\left(\mathrm{CD} 45^{+} \mathrm{CD}^{+}\right.$, red $)$in $\mathrm{mBM}(n=6)$, eBM 4 wk $(n=5)$, eBM 8 wk $(n=5)$ and intact mPB $(n=1)$. Error bars represent s.e.m.

Fig. 3. In vitro microfluidic culture of eBM within the bone marrow-on-achip. (a) Abundance of HSCs ( Lin $^{-} S c a 1^{+}{ }^{+} \mathrm{CKit}^{+}$cells, red) and hematopoietic progenitor cells (Lin`Sca ${ }^{+}$, cyan; Lin $^{-} \mathrm{KKit}^{+}$, purple; Lin $^{-} \mathrm{CD} 34^{+}$, green; $\operatorname{Lin}^{-} \mathrm{CD} 135^{+}$, blue) in mBM, femur bone marrow cultured for 4 and 7 days a stroma-supported culture system (mBM D4, mBM D7), fresh uncultured eBM, and eBM after 4 and 7 days of culture on-chip (eBM D4, eBM D7) ( $n=7$ for all conditions). (b) Abundance of long-term HSCs (Lin

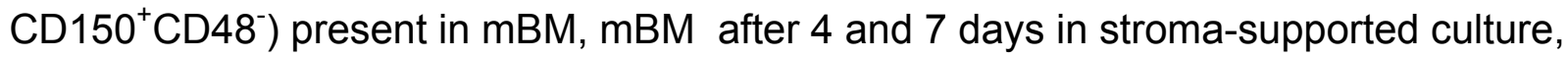
eBM and eBM after culture for 4 and 7 days $(n=7)$. Statistical analysis was conducted using two-tailed T-test assuming independent samples with equal variance. (c) Abundance of erythrocytes (Ter119 ${ }^{+}$, blue), myeloid cells $\left(\mathrm{CD} 45^{+} \mathrm{Mac} 1^{+/} \mathrm{Gr} 1^{+/-}\right.$, green), B cells $\left(\mathrm{CD} 45^{+} \mathrm{CD} 19^{+}\right.$, purple $)$and $\mathrm{T}$ cells $\left(\mathrm{CD} 45^{+} \mathrm{CD} 3^{+}\right.$, orange $)$in the $\mathrm{mBM}$ and eBM populations at the time of isolation compared to 4 and 7 days of culture $(n=6)$. (d) Abundance of HSCs and hematopoietic progenitor cells from fresh, uncultured eBM 
compared to 4 and 7 days of culture with and without supplemental cytokines (SCF, IL11, Flt-3, LDL) $(n=6)$. (e) The extent of bone marrow engraftment in lethally irradiated mice transplanted with $2.5 \times 10^{5} \mathrm{GFP}^{+}$cells from uncultured $\mathrm{mBM}$ or isolated from the eBM following 4 days of in vitro culture on-chip. Engraftment is presented as percentage of GFP+ cells in the lymphoid population $\left(C D 45^{+}\right)$of peripheral blood measured 6 weeks and 16 weeks after transplantation to confirm retention of functional hematopoietic progenitor cells and HSCs, respectively $(n=3)$. (f) Distribution of differentiated blood cells within the engrafted $\mathrm{CD} 45^{+}$population from $\mathrm{mBM}$ and eBM D4 transplants at 6 and 16 weeks after intravenous injection into lethally irradiated mice. Differentiated cell types include T cells $\left(\mathrm{CD} 45^{+} \mathrm{CD} 3^{+}\right.$, purple), B cells $\left(\mathrm{CD} 45^{+} \mathrm{CD} 19^{+}\right.$, green) and myeloid cells $\left(\mathrm{CD} 45^{+} \mathrm{Mac} 1^{+}\right.$, blue; $\mathrm{CD} 45^{+} \mathrm{Gr} 1^{+}$, red $)(n=3)$. Error bars represent s.e.m.

Fig. 4. Radiation toxicity of the bone marrow-on-a-chip. (a-d) Effects of $\gamma$ radiation ( 0,1 or $4 \mathrm{~Gy})$ on the abundance of $(\mathbf{a}) \mathrm{HSCs}\left(\mathrm{Lin}^{-} \mathrm{Sca} 1^{+}{ }^{\mathrm{CKit}}{ }^{+}\right),(\mathbf{b}, \mathbf{c})$ hematopoietic progenitors ( $\left.\mathrm{Lin}^{-} \mathrm{CD} 34^{+}, \mathrm{Lin}^{-} \mathrm{CKit}{ }^{+}\right),(\mathbf{d})$ lymphoid cells $\left(\mathrm{CD} 45^{+} \mathrm{CD} 3^{+}\right.$, $\left.\mathrm{CD} 45^{+} \mathrm{CD} 19^{+}\right)$and $(\mathbf{e})$ myeloid cells $\left(\mathrm{CD} 45^{+} \mathrm{Mac}^{+/-} \mathrm{Gr} 1^{+/-}\right)$within bone marrow of freshly isolated from femurs of living mice (in vivo), eBM cultured on-chip for 4 days (eBM), or 4 day old stroma-supported cultures (Dish) $n=5$ ). (f) Effect of G-CSF on the abundance of HSCs (Lin`Sca1 ${ }^{+}$CKit $^{+}$cells, black) and hematopoietic progenitor cells (Lin ${ }^{-} \mathrm{CKit}^{+}$, white; Lin ${ }^{-C D} 34^{+}$, gray) in eBM cultured on-chip for 4 days $(n=5)$. Statistical analysis was conducted using two-tailed T-test assuming independent samples with equal variance.. Error bars represent s.e.m.; $P$-values above individual bars represent 
(Revised NMETH-A19608)

comparisons between in vivo and experimental samples; brackets represent comparisons between experimental samples. 


\section{ONLINE METHODS}

Animals. CD1 mice were purchased from Charles River Laboratories and C57BL/6 mice and C57BL/6-Tg(UBC-GFP)30Scha/J mice were from Jackson Laboratories. All animal studies were reviewed and approved by the Animal Care and Use Committee of Children's Hospital Boston. For both implantation of devices and transplant experiments, no randomization or blinding was completed. All transplanted mice and those implanted with devices were equivalent (age, sex, strain).

Bone-inducing materials. Demineralized bone powder (DBP) was prepared from femurs harvested from CD-1 mice ${ }^{18}$. The femurs were washed in sterile water, extracted with absolute ethanol, and dehydrated with ether. The bones were crushed with a mortar and pestle and demineralized in $0.5 \mathrm{~N} \mathrm{HCl}(50 \mathrm{~mL} / \mathrm{g})$ for 3 hours at room temperature. After demineralization, DBP was washed with sterile water, extracted with absolute ethanol, dehydrated with ether, and was passed through a sieve with $250 \mu \mathrm{m}$ pores. $3 \mathrm{mg}$ DBP mixed with $30 \mu \mathrm{L}$ solution of type I collagen gel $(3 \mathrm{mg} / \mathrm{mL}$, Cellmatrix Type I-A, Nitta Gelatin Inc.), 100 ng BMP2 (Alpha Diagnostic Intl. Inc.), and 100 ng BMP4 (Alpha Diagnostic Intl. Inc.) was placed in the central cylindrical cavity (1 mm high $\times 4 \mathrm{~mm}$ diameter) of a device (1 $\mathrm{mm}$ high $\times 8 \mathrm{~mm}$ diameter) (Fig. 1A, B) which was fabricated from poly-dimethylsiloxane (PDMS) formed from prepolymer (Sylgard 184, Dow Corning) at a ratio of 10:1 base to curing agent and using biopsy punches (Miltex, Inc.). To seal the top of the central cylindrical cavity, a solid layer of PDMS (0.5 mm thick) and the device were bonded together using a plasma etcher (SPI Plasma-Perp II, Structure Probe, Inc.) in air for 30 seconds. 
Implantation of bone inducing materials. The PDMS devices filled with the bone-inducing materials were implanted subcutaneously on the backs of 8- to 12-weekold CD-1 mice or C57BL/6-Tg(UBC-GFP)30Scha/J mice, and harvested 4 or 8 weeks after implantation (Supplementary Fig. S1). Because implantation of devices was completed in all animals procured or bred, a randomization procedure was not performed. The PDMS device with two openings permitted access between the boneinducing materials and both the underlying muscle and overlying skin (Fig. 1A and Supplementary Fig. S1). The PDMS device with a single lower opening only permitted access to the muscle.

Histology and Immunohistochemistry. Engineered bone marrow (eBM) harvested from mice 8 weeks after implantation, and femurs from the same mice, were collected and fixed in $4 \%$ paraformaldehyde at $4^{\circ} \mathrm{C}$ for 24 hours. Tissues were transferred into $70 \%$ ethanol and stored at room temperature until processed. Tissues were embedded in paraffin and sectioned for subsequent staining hematoxalin and eosin (H\&E) staining, immunohistochemistry using anti-CXCL12 (SDF-1 beta; eBioscience, \#14-7991-83) or anti-CXCR4 (UMB2; Novus Biologicals LLC, \#NBP195362) polyclonal antibodies, followed by anti-rabbit IgG conjugated to HRP (Vector Laboratories). Slides were counterstained with hematoxylin. Tissues were also embedded in Tissue-Tek O.C.T. (Sakura Finetek USA, Inc.) and sectioned for subsequent immunofluorescence analysis using anti-CD31 (Abcom, \#ab28364), antiNestin (Abcam, \#ab6142), or anti-Leptin receptor (Abbiotec, \#250739), followed by antirabbit IgG conjugated to Alexa Fluor 488 (Life Technologies, \#A11034) or anti-mouse IgG conjugated to Alexa Fluor 488 (Life Technologies, \#A21202). 
Flow cytometry. To evaluate the distribution of the various hematopoietic cell populations, flow cytometric analysis was performed using 5-laser-Fortessa flow cytometer (Becton Dickinson). Cell types were evaluated based on expression of surface antigens that are characteristic for HSCs, long-term HSCs, hematopoietic progenitor cells and multiple differentiated blood cell lineages. The differential distribution was evaluated based on percentage of whole bone marrow. Because the HSCs and hematopoietic progenitor cell populations represent a small percentage of the total, representative flow cytometry plots are shown after excluding mature lineagerestricted ( Lin $\left.^{-}\right)$cells. The numbers shown on the FACS plots indicate the percent contribution relative to the whole bone marrow population. To harvest bone marrow cells, the eBM was removed from the PDMS devices, cut into small pieces and digested using $1 \mathrm{mg} / \mathrm{mL}$ collagenase (Roche) for $30 \mathrm{~min}$. Bone marrow cells harvested from eBM and normal mouse femur were stained in cold PBS containing $3 \%$ FBS and $0.05 \%$ sodium azide for 30 minutes with antibodies directed against 1) eFluor 450 hematopoietic lineage cocktail (1:5, eBioscience, \#88-7772-72), APC Sca1 (1:333, eBioscience, \#17-5981-82, clone D7), APC-eFluor780 cKit (1:160, eBioscience, \#471172-82, clone ACK2), FITC CD34 (1:50, eBioscience, \#11-0341-82, clone RAM34) or APC CD34 (1:10, eBioscience, \#50-0341-82, clone RAM34), and PE CD135 (1:20, eBioscience, 12-1351-82, clone A2F10) to identify HSCs (Lin-Sca ${ }^{+}{ }^{-} \mathrm{CKit}^{+}$), progenitor cells (Lin`Sca1 ${ }^{+}$, Lin $\left.\left.^{-} \mathrm{CKit}^{+}, \mathrm{Lin}^{-} \mathrm{CD} 34^{+}, \mathrm{Lin}^{-} \mathrm{CD} 135^{+}\right), 2\right)$ lineage cocktail $(1: 5$, eBioscience, \#88-7772-72), PE CD150 (1:40, eBioscience, \#12-1501-82, clone 9D1), and APC CD48 (1:160, eBioscience, \#17-0481-82, clone HM48-1) to identify long-term HSCs (Lin CD150 ${ }^{+}$CD48 $^{-}$), or 3) APC-eFluor780 Ter119 (1:40, eBioscience, \#47-5921-82, clone 
TER-119), Pacific Blue CD45 (1:200, eBioscience, \#48-0451-82, clone 30-F11), APCCy7 CD3 (1:20, BD Pharmingen, \#560590, clone 17A2), APC CD19 (1:80, BD

Pharmingen, \#550991, clone 1D2), APC-Cy7 Mac1 (1:160, eBioscience, \#47-0112-82, clone M1/70), and APC Gr1 (1:160, eBioscience, \#17-5931-82, clone RB6-8C5) to identify erythrocytes (Ter119), leukocytes (CD45), B cells (CD19), T cells (CD3), and myeloid cells (Mac1 and Gr1). Cellular viability was evaluated using propidium iodide (25 ng/mL, Millipore).

In vitro microfluidic culture on-chip. The cylindrical eBM was removed from the PDMS device, pierced multiple (4-6) times with a surgical needle (32 gauge), and cultured in a similarly shaped central chamber within a microfluidic chip device (Fig. 1A, B) that was separated from overlying and underlying microfluidic channels $(200 \mu \mathrm{m}$ high) by porous PDMS membranes (20 $\mu \mathrm{m}$ thick with $100 \mu \mathrm{m}$ pores). The microfluidic channels were molded against master molds made by standard photolithography using the negative-photoresist SU-8 (MicroChem Co.). PDMS membranes were made by spin-coating a PDMS layer on a silanized glass slide at $1500 \mathrm{rpm}$ for 60 seconds and then curing in an $80^{\circ} \mathrm{C}$ oven for at least 2 hours. An array of $100 \mu \mathrm{m}$ pores with a 100 $\mu \mathrm{m}$ pitch was made on a PDMS membrane using a laser cutter (Versal Laser VL-300, Universal Laser Systems, Inc.). Microfluidic channel layers and porous PDMS membranes were bonded together using a plasma etcher in air for 30 seconds. To allow introduction of solution into the channels, access holes were punched through the top channel layer with a $2 \mathrm{~mm}$ biopsy punch and the inlets and the outlets were connected with tubes (i.d. $=1 / 32$ inch). The microfluidic device was oxidized using a plasma etcher in air for 10 min to make the PDMS surface hydrophilic. The eBM was 
inserted into the central chamber (which was bonded to the bottom channel layer) before attachment of the top channel layer. The microfluidic device was placed between two acrylic plates ( $3 \mathrm{~mm}$ diameter) made by a laser cutter and immobilized using screws (Fig. 1B) To maintain cellular viability of the eBM, culture medium (SFEM basal medium, StemCell Technologies) containing cytokines ${ }^{33}$ (50 ng/mL mouse SCF, $100 \mathrm{ng} / \mathrm{mL}$ mouse IL-11, $100 \mathrm{ng} / \mathrm{mL}$ mouse FLt-3, and $20 \mu \mathrm{g} / \mathrm{mL}$ human LDL, StemCell Technologies) was perfused through the top and bottom channels ( $1 \mu \mathrm{L} / \mathrm{min}, 0.005$ dyn $/ \mathrm{cm}^{2}$ ) using a syringe pump (BS-8000, Braintree Scientific, Inc.).

Stroma-supported bone marrow cell culture. Bone marrow stromal cells were harvested from 8- to 12-week-old C57BL/6 mice, re-suspended in DMEM medium (Gibco) containing 20\% FBS (Gibco), GlutaMAX (Gibco), and 100 units/mL penicillinstreptomycin (Gibco), and cultured in the same medium on tissue culture plates (Falcon), changing the medium every other day to create stromal feeder layers. After the adherent monolayer became established (about 3 weeks), the cells were irradiated with 12 Gy. Bone marrow cells harvested from femurs of C57BL/6- $\operatorname{Tg}(\mathrm{UBC}$ GFP)30Scha/J mice were cultured on this bone marrow stromal cell layer using the same culture medium as use in the microfluidic culture.

Bone marrow transplantation. Bone marrow transplantation was performed on 8-week-old C57BL/6 mice exposed to two doses of radiation measuring 6 Gy separated by 2-3 hours. Because these mice were purchased from a vendor and randomized upon arrival, a randomization procedure was not conducted. The bone marrow cells were harvested from femurs of C57BL/6-Tg(UBC-GFP)30Scha/J mice or from eBM produced in similar GFP-labeled mice after 4 days of microfluidic culture. $2.5 \times 10^{5}$ bone marrow 
cells were delivered by intravenous (i.v.) tail vein injection within 12 hours of lethal irradiation. Engraftment was measured 6 weeks and 16 weeks after transplant using retro-orbital bleeds and flow cytometric analyses.

Micro-computed tomographic (micro-CT) analysis. eBM harvested from mice 4 and 8 weeks after device implantation were fixed for 48 hours in $4 \%$ paraformaldehyde and stored in $70 \%$ ethanol at $4{ }^{\circ} \mathrm{C}$. Vertebrae harvested from the same mice immediately device removal were handled similarly. Both the eBM and vertebrae were imaged (in 70\% ethanol) with an XRA-002 X-Tek MicroCT system. X-ray transmission images were acquired at $55 \mathrm{kV}$ and $200 \mu \mathrm{A}$ and the $3 \mathrm{D}$ reconstructions were performed using CT-Pro (Nikon Metrology); surface renderings were generated using VGStudio Max.

\section{Compositional backscattered scanning electron (BSE) micrographs and} elemental mapping. eBM and vertebrae harvested and fixed as described for microCT were serially dehydrated into $100 \%$ ethanol and then embedded in Spurr's resin and sectioned at the desired imaging plane using a slow speed diamond saw. The resulting sections were polished with silicon carbide papers down to P1200, sputter coated with gold and examined using a Tescan Vega-3 scanning electron microscope equipped with a Bruker X-Flash 530 energy dispersive spectrometer (EDS). All EDS spectra and elemental maps were acquired at $20 \mathrm{keV}$ accelerator voltage. For calculating elemental composition of both the sectioned implant and vertebra samples, 10 point spectra from the surface of each sample were acquired and the percent phosphorus and calcium content was determined by averaging the obtained values \pm s.e.m. 
$\gamma$-Radiation. Freshly harvested eBM made in C57BL/6-Tg(UBC-GFP)30Scha/J (Jackson Laboratories) mice, $1 \times 10^{7}$ mouse femur bone marrow cells maintained in stroma-supported culture, and 8 week old C57BL/6-Tg(UBC-GFP)30Scha/J were exposed to one does of gamma-irradiation (Cs-137), 1Gy or 4Gy. Ninety-six hours after irradiation, marrow from the eBM cultured on-chip, bone marrow in stroma-supported culture, and bone marrow from the femurs of live mice were collected for flow cytometric analysis. $500 \mathrm{U} / \mathrm{mL}$ granulocyte colony-stimulating factor (G-CSF, Sigma-Aldrich) was added in the culture medium containing cytokines 24 hours after exposure to gammairradiation. After 72 hours in culture on-chip with G-CSF, marrow from the eBM was collected for flow cytometric analysis.

Statistics. Sample size for in vitro and in vivo experiments was determined based on a minimum of $n=3$ biological replicates. Statistical differences were analyzed by Student's T-test. All statistical evaluation was conducted using a two-tailed T-test, assuming independent samples of normal distribution with equal variance. $P<0.05$ were deemed statistically significant; all error bars indicate standard error of the mean.

Editorial summary:

Bone marrow formed in a cylindrical PDMS device implanted in a mouse can be surgically removed and cultured for a week in vitro without losing any of the hallmarks of in vivo bone marrow niches. 